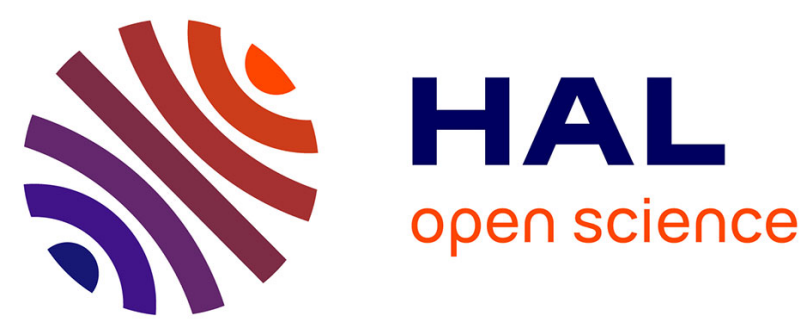

\title{
Power optimization in two-tier networks
}

\author{
Seifeddine Bouallegue, Kaouthar Sethom, Guy Pujolle
}

\section{To cite this version:}

Seifeddine Bouallegue, Kaouthar Sethom, Guy Pujolle. Power optimization in two-tier networks. International Conference on Computer, Communications, and Control Technology (I4CT), 2015, Apr 2015, Kuching, Malaysia. pp.565-569, 10.1109/I4CT.2015.7219642 . hal-01202355

\section{HAL Id: hal-01202355 https://hal.science/hal-01202355}

Submitted on 4 Oct 2015

HAL is a multi-disciplinary open access archive for the deposit and dissemination of scientific research documents, whether they are published or not. The documents may come from teaching and research institutions in France or abroad, or from public or private research centers.
L'archive ouverte pluridisciplinaire HAL, est destinée au dépôt et à la diffusion de documents scientifiques de niveau recherche, publiés ou non, émanant des établissements d'enseignement et de recherche français ou étrangers, des laboratoires publics ou privés. 


\section{Power Optimization in Two-tier Networks}

\author{
Seifeddine Bouallegue, \\ INNOV'COM Lab, SUPCOM \\ University of Carthage \\ Tunisia
}

\author{
Kaouthar Sethom \\ ENICarthage, National \\ Engineering school of Carthage \\ Tunisia
}

\author{
Guy Pujolle \\ LIP6, University of Paris 6 \\ France
}

\begin{abstract}
A future cellular network demands a new architecture that can facilitate the autonomous deployment of the infrastructure similar to Wi-Fi. A novel method of deploying a cellular network has been introduced with small cellular base stations, or femtocells, autonomously deployed by users in an underlay macro-cellular network. This autonomous cellular concept allows the network to grow in an organic manner, which reduces the traditional work on infrastructure installation, network configuration and maintenance. To this end, we propose hereafter an intelligent and autonomic system for power control in the femtocell context.

The first simulation results seem to confirm the advantage of our solution in reducing energy consumption.
\end{abstract}

Keywords: Macro, microcell, LTE, mobility management.

\section{INTRODUCTION}

Femtocell networks are unique in that they are largely installed by customers or private enterprises often in an ad hoc manner without traditional RF planning, site selection, deployment and maintenance by the operator. Moreover, as the number of femtocells is expected to be orders of magnitude greater than macrocells, manual network deployment and maintenance is simply not scalable in a cost-effective manner for large femtocell deployments.

Femtocells must therefore support an essentially plug-and play operation, with automatic configuration and network adaptation. In the LTE overall description specification [1], the self-configuration process is defined as the process where the newly deployed base stations are configured by automatic installation procedures to get basic parameters and download necessary software for operation. In this paper, we propose a new energy-aware handoff algorithm that tries to adjust femtocell power with the regards to unwanted mobility events.

There are several reasons for the growing awareness of energy-efficient wireless networks in the telecommunication community. Increasing energy prices imply that electricity bills have become a significant cost factor for mobile operators. In addition, mobile telephony and mobile broadband are entering new emerging markets, with an increasing share of base stations that are not connected to the electricity grid. Such off-grid sites are typically diesel powered, where fuel is costly and distribution often unreliable for distant sites that are difficult to access. For operators with many off-grid sites, energy provision may contribute up to 50 percent of their total operational cost. There is also an increasing awareness in the society about manmade climate effects, and the need to slow down global warming. Moreover, political initiatives are beginning to put requirements on manufacturer and operators to lower $\mathrm{CO} 2$ emissions of communication networks.

The rest of the paper is organized as follows: section 2 describes state of the art on energy management in wireless networks. Section 3 presents our algorithm for femtocell power adjustement. Section 4 and 5 try to evaluate our proposal through an analytical model and simulations.

\section{STATE OF THE ART}

The transmit power of femtocell consists of: (i) pilot power (responsible for cell selection and channel estimation) and, (ii) traffic power (includes signalling power and data power). Pilot power determines the coverage of the cell: large pilot power results in a large cell coverage and small pilot power may lead to insufficient coverage. Moreover the larger the pilot power the less the power left for traffic, which result in minimizing the throughput of the femtocell.

The related work in the literature on cellular power optimization mostly deals with centralized computation methods. Such mechanisms involve the calculation of parameters such as number and locations of base stations (BS), pilot channel transmit powers or antenna configurations using a central server running an optimization algorithm. The configuration statistics of the whole network are passed on to the central entity, which then computes the optimal/quasi-optimal configuration settings. The algorithms are Genetic Algorithms, Greedy Algorithms, Simulated Annealing or other classical optimization methods [2-6]. 


\section{POWER SAVING AlgOrithm}

We consider an overlay wireless network with two layers: macrocell and femtocell. During operation, information on the number of mobility events and their time are collected and used for further refinement of the coverage by self-optimization of the pilot power of the femtocell. We here propose an optimization method that takes decision based on unwanted mobility events from transient users into account that briefly hand over to the femtocell and handout immediately after passing.

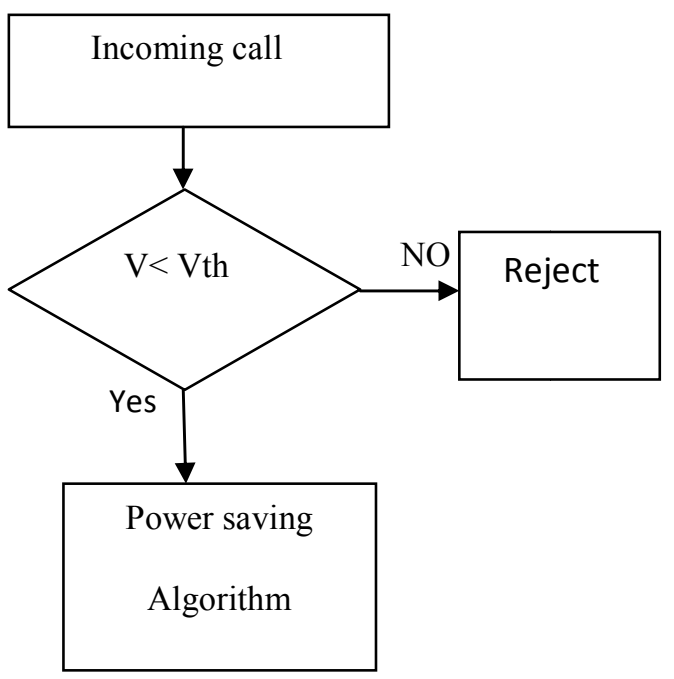

Figure 1: Mobility Management Scenario

We hereafter give a brief overview of the system model where a number of mobile users are deployed randomly under the coverage of the macro BS with varied velocity and undergoing calls of varied service type:

1. No hanfdoff is performed from macro to femtocell within ' $R$ ' meter of radius of the macro base station because the SINR of mobile within this area is assumed to be quite high.

2. When the mobile user is moving at a very high velocity, no handoff from the macro to the femto is executed. The mobile remains attached to the Macrocell. As a user with very high velocity it undergoes huge number of macro/femto handoff within a very short period of time. The overhead of the macro BS thus increases unnecessarily. Hence, in this paper we have considered a velocity threshold Vth (see section 4).

The general principle of the proposed scheme is depicted in Figure 1.

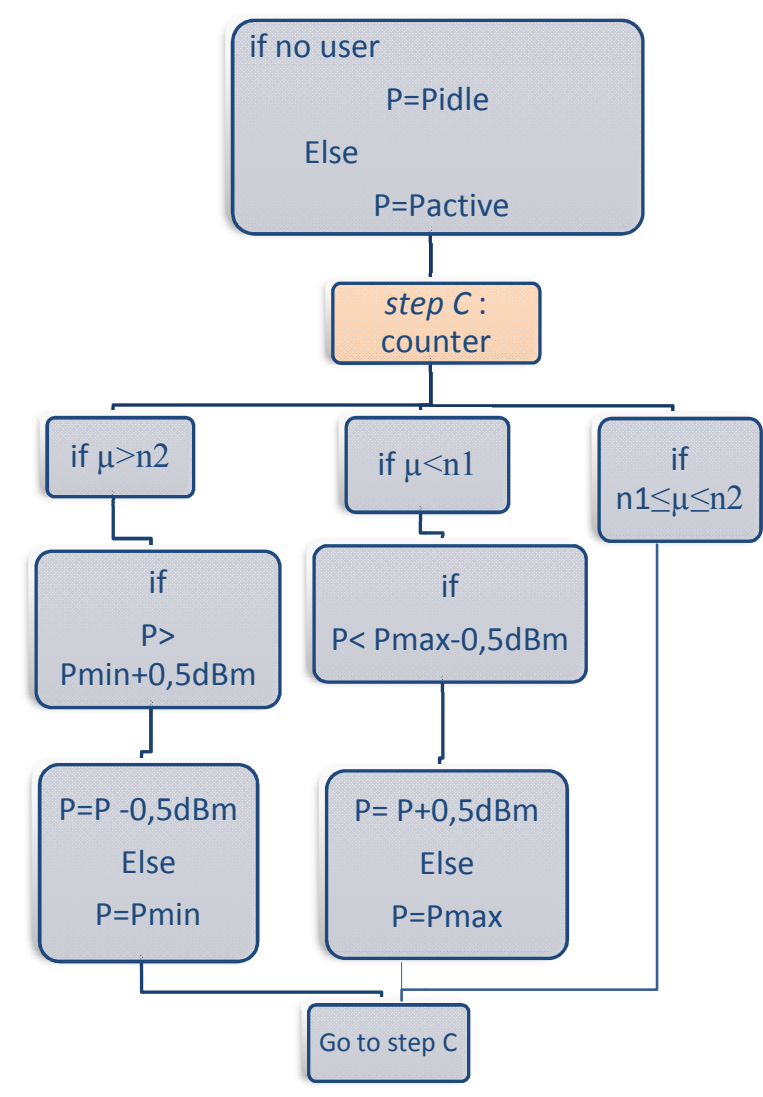

Figure 2: Power Saving Algorithm Flow Chart

\section{A. Algorithm}

\section{1) Initialization phase}

Initially, the femtocells are considered to be in the idle mode with all the pilot transmissions and associated radio processing at lower power sniffer (Eidle). The femtocell becomes active (Eactive) only when any user is active under its coverage. This idle/active status aims to enhancing the energy consumption.

\section{2) Optimization phase}

We define two priority levels: the highest one U1 for the FAP owner and U2 for any other guest user.

The algorithm follows Claussen and al. [7] method. It eliminates unwanted mobility events from transient users that briefly hand-in to the femtocell and hand-out immediately after passing the femto:

i. If the number of unwanted mobility events of passing users exceeds a pre-defined value of $n 1$ events per time $t 1$ the femto must reduce its pilot power by - 
$0,5 \mathrm{dBm}$. However, the minimum FAP power should cover U1 user if he is attached to his femtocell.

ii. The femtocell then starts a new mobility event count for the updated configuration. If the number of unwanted events is smaller or equal to $n 2$ events for a time period $t 2$, this is a stability phase, no power modification.

iii. Finally, if the number of unwanted events is bigger to $n 2$ for a time $t 2$, the femtocell increases its pilot power by $+0,5 \mathrm{dBm}$, to provide improved indoor coverage. The maximum Power is limited by an upper value Pmax to avoid inter-femtocell interference.

This means that at the initial stage:

(1)

$$
\text { Emin }=\text { Eidle, and Emax= Eactive }
$$

However, at the optimization stage:

\section{If U1exists}

$\mathrm{Emin}=\mathrm{E}($ just to cover $\mathrm{U} 1)$

Else

$$
\text { Emin= Eidle, }
$$

In case of power increasing scenario, the Femtocell $\mathrm{j}$ must measure the level of other-cell interference I that is received from its vicinity $(V)$ as it's illustrated in the figure below.

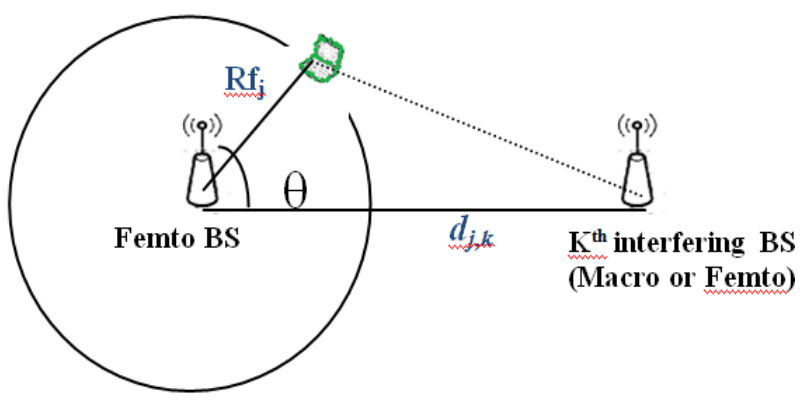

Figure 3: Interference Evaluation Scheme

The interfering signal received at the edge border of the cell at the ith iteration is expressed by:

(3)

$$
\begin{gathered}
I^{i}(R j)=I_{\text {macro }}+ \\
\sum_{k} \frac{P^{i} k}{A_{s} L_{w}\left(R j^{2}+\mathrm{d}_{\mathrm{j}, \mathrm{k}}{ }^{2}-2 \mathrm{Rjd}_{\mathrm{j}, \mathrm{k}} \cos \theta_{\mathrm{k}}\right)^{-\alpha / 2}}
\end{gathered}
$$

Where:

$R j$ is the current femtcell radius, $\mathrm{d}_{\mathrm{j}, \mathrm{k}}$ is the distance between the Femto $\mathrm{j}$ and the Femto $\mathrm{k}, I_{\text {macro }}$ is the interfering signal from the macrocell,$P^{i} k$ is the surrounding femtocells $\mathrm{k}$ transmit power and, $\alpha, A_{s}, L_{w}$ are the path loss exponents and the wall penetration loss.

The femtocell $\mathrm{j}$ evaluates the received interference plusnoise power and Pmax is calculated so that:

$$
10 \log I^{i}(R j)+W<\gamma t h j
$$

Where,

$\gamma$ thj is predifined threshold for tolerate interference level at femtocell $\mathrm{j}$ and $\mathrm{W}$ is the noise.

\section{ANALYTICAL MODEL}

In this section, a simple analytic model is developed to mainly analyze the performance parameters of the proposed scheme.

Let's first begin by defining the speed upper bound for our proposal. Given a mobile user UE with an average speed $v$ and traversing a two-tier network (for a distance $\Delta)$, a valid handover occurs if: the time that the mobile will spend in the femtocell is long enough to compensate for the data loss due to the switching overhead. This means that:

$$
v<v_{\text {th }}=\frac{\Delta\left(R_{\text {femto }}-R_{\text {macro }}-\rho\right)}{\left(R_{\text {femto }}-\rho\right) T_{h}}
$$

Where:

$T_{h}$ is the handover delay,

$R_{\text {femto }}$ is the throughput of the femtocell and $R_{\text {macro }}$ is the trouhput of the macro.

$\rho$ is a hysteresis factor introduced to avoid handover when the two competing cells have negligible bandwidth difference.

In our proposal, the active state is divided into 3 cases:

1. The number of unwanted handoff $k$ is under $n l$ in which case the femtocell Power $P$ has to be increased by $+0,5 \mathrm{dBm}$. With the respect of an upper power limit Pmax.

2. The number of unwanted handoff is $n 1 \leq k \leq n 2$ in which case no change in femtocell Power (so $E$ is set to Ecurrent)

3. The number of unwanted handoff is $k>n 2$ in which case $\mathrm{P}$ has to be decreased by $-0,5 \mathrm{dBm}$ (with respect 
to an under power limit that correspond to the idle state Pidle)

Let's now try to calculate the probability of an unwanted handoff.

In this work, the $P(k, T)$ denotes the probability of $k$ handover events occurring within the period time $T$. In general, the Poisson distribution is represented as

$$
P(k, T)=\frac{(\lambda T)^{k}}{k !} e^{-\lambda T}
$$

Where $\lambda$ is the average proportion of the event happened. The probability of a handover which has not occurred within time $T_{j}$ is $P\left(0, T_{j}\right)=e^{-\lambda_{j} T_{j}}$, Where, $\lambda_{j}=\frac{1}{t_{j}}$ and $t j$ is the time period of the received SNR of Femtocellj (FBSj) from the UE is larger than the threshold value. The $\lambda \mathrm{jTj}$ is the average handover number within the time duration $\mathrm{Tj}$.

The probability of handover is then:

$$
1-e^{-\lambda_{j} T_{j}}
$$

The average energy consumption for the data transmission of a UEj (user equipement) performing a handover in the Ashraf protocol [7] is:

$$
E_{A}(t)=e^{-\lambda j t} * E_{i \mathrm{~d} l e}+\left(1-e^{-\lambda j t}\right) * E_{\text {active }}
$$

Where $E_{0}$ is the average energy consumption of the data transmission of an UE before entering the coverage of FBSj.

We introduce two values of tdwell and texpected, where tdwell is the dwell time of a UE of keeping the communication with FBSj, where the UE is in the coverage of FBSj and texpected is the expected transmission time of data of the UE. If a UE cannot completely transmit all of the data within the tdwell, then an unwanted handoff occurs.

The probability of a wanted handover is when the remainder data of UEj can be completely uploaded within time texpectedj through the FBS i.e. no handover within texpectedj:

$$
P\left(0, t_{\text {expected }_{k}}\right)=e^{-\widehat{\lambda}_{k} t_{\text {expected }_{k}}}
$$

where the $\hat{\lambda}_{k}=\frac{1}{t_{\mathrm{dwellk}}}$ is the average number of complete uploading within time tdwell $_{k}$, where texpected $_{k}$ is the average expected transmission time using $\mathrm{FBS}_{\mathrm{k}}$ and tdwell $\mathrm{l}_{\mathrm{k}}$ is the time starting from the UE communicating with $\mathrm{FBS}_{\mathrm{k}}$ and stopping to communicate with $\mathrm{FBS}_{\mathrm{k}}$.

The expected transmission time can be calculated as:

$$
\mathrm{t}_{\text {expected } \mathrm{k}}=\frac{\text { Dremainder }_{\mathrm{k}}}{\mathrm{R}_{\mathrm{k}}}
$$

Where, Dremainder ${ }_{k}$ is the remainder data and $\mathrm{R}_{\mathrm{k}}$ the average transmission rate of $\mathrm{FBS}_{\mathrm{k}}$.

At initial time $\mathrm{t}=0$ : The femtocell energy can be Ecurrent $=$ Eidle if no attached user to the femto else Ecurrent=Eactive

$$
\begin{aligned}
E_{S}=E_{0}+\frac{1}{n} \sum_{j} & e^{-\lambda j T j} * E_{i \mathrm{~d} l e} \\
& +\frac{1}{n} \sum_{j}(1 \\
& \left.-e^{-\lambda j T j}\right)\left(\left(1-e^{-\widehat{\lambda \jmath} t \mathrm{dwell} j}\right)\right. \\
& \left.* E_{\text {current }}+e^{-\widehat{\lambda \jmath} t \text { dwell } j} * E_{\text {opt }}\right)
\end{aligned}
$$

Where:

$$
\begin{aligned}
& \text { If } j<n 1 \\
& \mathrm{E}_{\mathrm{opt}}=\left\{\begin{array}{c}
E(X=\text { Pcurrent }+j * o, 5) \text { if } X \leq P \max \\
\text { Else } \\
\text { Emax }=E \text { active }
\end{array}\right.
\end{aligned}
$$

If $n 1=<j<n 2$

$$
\mathrm{E}_{\mathrm{opt}}=\text { Ecurrent }
$$

If $j>n 2$

$$
\mathrm{E}_{\mathrm{opt}}=\left\{\begin{array}{c}
E(X=\text { Pcurrent }-j * 0,5) \text { if } X \geq P \min \\
\text { Else } \\
\text { Emin }
\end{array}\right.
$$

It is easily seen that $E-E_{S} \geq 0$; since $E_{\text {current }} \leq$ Eactive and $E_{\text {opt }} \leq$ Eactive.

Our proposal outperforms a classical approach in terms of energy consumption. 


\section{SIMULATION RESULTS}

In this section, simulation results are presented to evaluate the performances of the proposed scheme in LTE networks with femtocells. For comparison purpose, the performances of the network with Ashraf solution [7] are also presented.

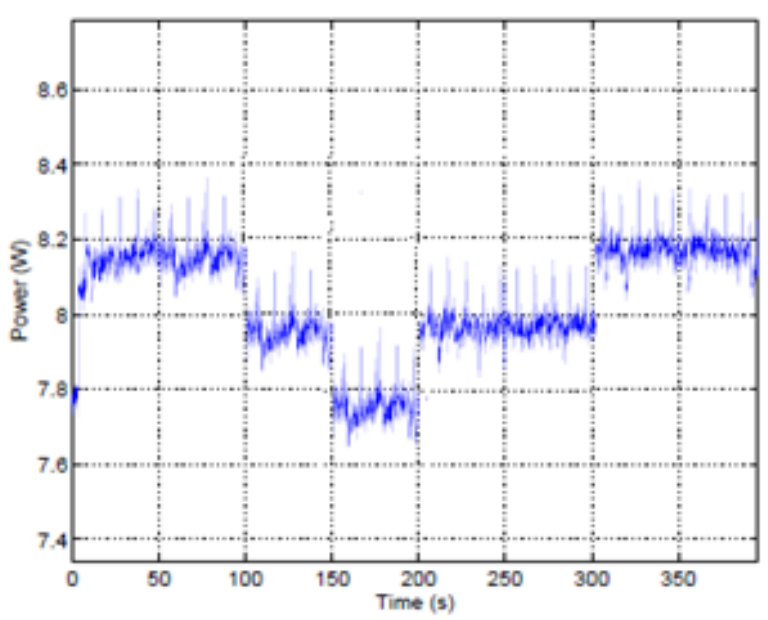

Figure 4: Handover blocking probability

Ashraf et al. [7] recently proposed an energy-saving procedure that allows the femtocell to completely switch off its radio transmissions and associated processing when not involved in an active call. From our point of view, this method is not realistic because wake up will be done each time a new user come, which will be energy consuming and will increase the ping pong effects in the network.

Figure 4 shows that our algorithm reduces handover blocking probability because the network coverage is optimized in order to eliminate unwanted mobility events.

Figure 5 confirms that our proposal reduces considerably the amount of energy consumption (the gain is more than $25 \%$ in a heavy network). These simulations show the power of a femtocell implementing our dynamic power allocation algorithm. The power is auto-configured, then auto-optimized according to users' mobility. We can see that femtocells have different coverage due to different optimized transmitting power. Our proposal is more context-aware then previous works. Moreover, it decrease holes in the network compared to [7].

\section{CONCLUSION}

In this paper, we have proposed an energy efficient mobility based algorithm for reducing unnecessary handoff in hierarchical macro/femto networks while balancing the load of macro and the femto BSs at minimal energy consumption. The performance of the proposed algorithm is also analyzed using an analytical model. Simulations show good performances.

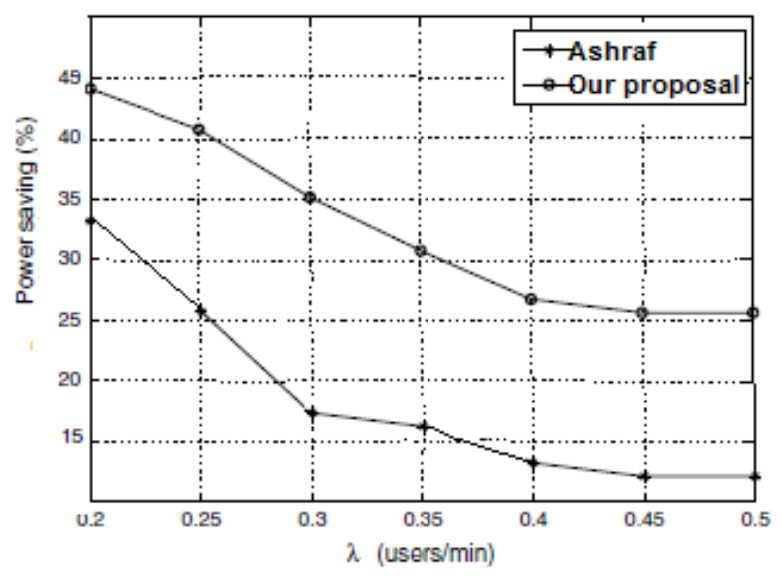

Figure 5: Network energy consumption

\section{REFERENCES}

[1] R. Kim, J. S. Kwak, and K. Etemad, "WiMAX femtocell: requirements, challenges, and solutions," IEEE

Communications Magazine, vol. 47, no. 9, pp. 84 -91,, Sep. 2009.

[2]L. T. W. Ho and H. Claussen. "Effects of user-deployed, cochannel femtocells on the call drop probability in a residential scenario". IEEE PIMRC 2007.

[3] K. Lieska, E. Laitinen, J. Lahteenmaki, "Radio Coverage Optimization with Genetic Algorithms," IEEE PIMRC 1998.

[4] A. Molina, G.E. Athanasiadou, A.R. Nix, "The Automatic Location of Base-Stations for Optimised Cellular Coverage: A New Combinatorial Approach," IEEE VTC 1999.

[5] I. Siomina, P. Varbrand, D. Yuan, "Automated Optimization of Service Coverage and Base Station Antenna Configuration in UMTS Networks," IEEE Wireless Communications, vol. 13, no. 6, pp. 16-25, Dec 2006.

[6] D. Fagen, P.A. Vicharelli, J. Weitzen, "Automated Coverage Optimization with Controlled Overlap," IEEE Transactions on Vehicular Technology, vol. 57, no. 4, pp. 2395, Jul 2008.

[7] H. Claussen, F. Pivit, L.T. W. Ho, "Self-Optimization of femtocell coverage to minimize the increase in core network mobility signalling", Bell Labs Technical Journal, Vol 14, issue 2, 2009.

[8] "A seamless handover scheme in IPv6-based mobile networks", $\mathrm{H}$. Oh,K. Yoo, J. Na, C. Kim, International Journal of Ad Hoc and Ubiquitous Computing 4 (1) (2010) 54-60.

[9] "Novel handoff decision algorithm in hierarchical Macro/Femto-cell networks", J.M. Moon, D.H. Cho, IEEE WCNC 2010, Sydney, Australia, 2010, pp.1-6. 